Military Technical College Kobry El-Kobba Cairo, Egypt

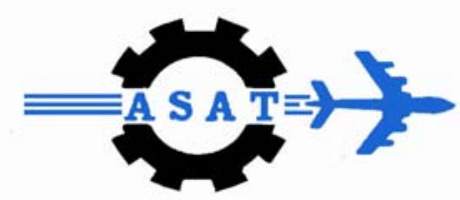

12-th International Conference on Aerospace Sciences \& Aviation Technology

\title{
FINITE ELEMENT ANALYSIS OF COMPRESSOR BLADES UNDER EXTENSION, BENDING AND TORSION LOADS
}

\author{
PART I: ISOTROPIC MATERIALS
}

\author{
A. M. FARID* and M. ADNAN ELSHAFEI
}

\begin{abstract}
A finite element formulation is presented for modeling the dynamic as well as static response of a compressor blades subjected to extension, bending and torsional loads. The formulation is derived from the variational principle with the consideration for the total potential energy of the structure based on the classical lamination theory. The compressor blade is modeled as an advanced beam with idealized rectangular cross-section that takes the warping effect into consideration. The bending-torsion coupling is introduced in the stiffness and mass matrices. A one dimensional linear isoparametric element with hermit cubic shape function is used. A two end nodes and one intermediate node as well is implemented for the finite element formulation. A Matlab interactive code was developed to solve a blade with a multi action loads. The results obtained are compared to the available analytical and finite element results.
\end{abstract}

\section{KEYWORDS}

Finite Element, compressor blade design, structural analysis, isotropic materials, solid mechanics. 


\section{NOMENCALUTRE}

\begin{tabular}{|c|c|}
\hline$\varepsilon$ & Axial strain. \\
\hline$\gamma$ & ... shear strain. \\
\hline$\varphi(\mathrm{x})$ & ... The twist angle at each section. \\
\hline$\theta$ & ... The bending angle (slope). \\
\hline$\rho$ & ... Density. \\
\hline$\omega$ & ... Natural frequency of the vibration. \\
\hline$v$ & ... Poisson's ratio. \\
\hline$\lambda$ & ... The Warping function. \\
\hline$\varphi_{1}, \varphi_{2}, \varphi_{3}$ & ... Torsion nodal displacements. \\
\hline$\Delta(\lambda)$ & ... polynomial of degree $n$ in $\lambda$. \\
\hline$\Omega(x)$ & ... Constant distributed load acting on beam element. \\
\hline a & ... Height of cross section of the beam. \\
\hline b & ... Width of cross section of the beam. \\
\hline $\mathrm{C}_{1}, \mathrm{C}_{2}, \mathrm{C}_{3}, \mathrm{C}_{4}$ & ... Constants of integration. \\
\hline $\mathrm{f}_{\text {axial }}(\mathrm{x})$ & ... Axial shape functions. \\
\hline $\mathrm{f}_{\text {bending }}(\mathrm{x})$ & ... Bending shape functions. \\
\hline $\mathrm{f}_{\text {torsion }}(\mathrm{x})$ & ... Torsion shape functions. \\
\hline$f_{\text {Axial-Warping }}$ & ... The effect of warping on torsional shape functions. \\
\hline$h$ & ... Single Element length. \\
\hline$m(x)$ & ... Mass per unit length. \\
\hline $\mathrm{m}_{\text {axial }}$ & ... Axial mass matrix. \\
\hline $\mathrm{m}_{\text {bending }}$ & ... Bending mass matrix. \\
\hline $\mathrm{m}_{\text {torsion }}$ & ...Torsion mass matrix. \\
\hline $\mathrm{n}$ & ... Number of elements. \\
\hline $\mathrm{u}_{1}, \mathrm{u}_{2}$ & ... Axial nodal displacements. \\
\hline$w_{1}, \theta_{1}, w_{2}, \theta_{2}$ & ... Bending nodal displacements. \\
\hline V & ... Volume. \\
\hline A & ... Cross sectional area $(A=a \cdot b)$. \\
\hline $\mathrm{D}$ & ... Dynamic matrix. \\
\hline$E$ & ... Modulus of Elasticity. \\
\hline $\mathrm{F}$ & ... Total mechanical Loads on single beam element. \\
\hline$F_{X}$ warping & $\ldots$ The effect of torsional warping on torsional stiffness. \\
\hline G & ... Shear modulus. \\
\hline$I_{m}(x)$ & ... Mass polar moment of inertia per unit length. \\
\hline l & ... Second area moment of inertia. \\
\hline $\mathrm{J}$ & ... Area Polar moment of inertia. \\
\hline$[\mathrm{K}]$ & ... Stiffness matrix. \\
\hline $\mathrm{K}_{\text {axial }}$ & ... Axial stiffness matrix. \\
\hline K bending & ... Bending stiffness matrix. \\
\hline Ktorsion & ...Torsion stiffness matrix. \\
\hline$K_{\text {warping }}$ & ... Cross-sectional warping coefficient. \\
\hline L & ... Length of the Cantilever beam. \\
\hline$P(x)$ & ... Constant axial force acting on beam element. \\
\hline $\mathrm{T}(\mathrm{x})$ & ... Constant Torque moment acting on beam element. \\
\hline$U_{i}$ & ... Strain energy of the total system. \\
\hline$W_{e}$ & ... External work done on the system. \\
\hline
\end{tabular}




\section{INTRODUCTION}

The failure of turbine and compressor blades due to multi mechanical applied loads, poor handling and intolerable noise has led to an increased interest in the study of the static and dynamic response of these blades. To improve the dynamic and static performance of these blades during their operations, a model must be considered and studied concerning with problems and loads they are subjected to. The compressor and turbine blades are undergo extension, bending and torsional loads, while an assumption must be consider, that the rotor must be operating at steady state conditions such that the blade fixation could be considered as fixed end cantilever beams. Besides the distribution of bending and torsion loads are constant along the length of the blades unlike the real case where the pressure loads on the blade span.

Several researches have studied the calculation of the uncoupled modes of arbitrarily shaped cantilever beams has been extensively investigated [1-4], but little work has as yet been done on calculating the coupled modes of such beams. The modeling of beam structures with coupled behavior presented in [5-11]. Sakawa and Luo [6] used a shear-indeformable theory to model a mass coupled beam. The internal beam damping was included in their model, the beam was mounted on a rotating shaft and an actuation torque was provided to the shaft by a motor. Banks and Smith [7] studied a coupling problem similar to that of Sakawa and Luo; however, in their model the warping effects and the internal shear damping were considered. Shen [8] also employed a shear-indeformable theory in which the warping effect was not included in composite beams. Banerjee and Williams [9] studied the vibration in a beam with geometrical coupling. Despite they used the shear-deformable theory, warping effect was ignored. A shear deformable theory is simplified by Sankar [11] to be applicable for a one-dimensional beam analysis. The warping effects caused by St. Venant torsion and warping effect are explicitly included in his model. Sankar theory shows some results identical to those achieved by Boresi et al [10]. A quite good agreement for a laminate beam is obtained between Sankar results and those reported by Tsai et al [13].

Therefore, the objective of this investigation is to develop a finite element formulation for modeling the static and dynamic response of a compressor blade simplified as an advanced cantilever beam subjected to axial, bending and torsion loads. The warping effect due to the torsion is taken into consideration during the analysis, and the bending-torsion coupling is introduced in stiffness and mass matrices. The equation of motion is obtained via the principle of total potential energy. A finite element interactive code, designated as "ABTIB" is developed on the basis of the analysis during this investigation. Numerical examples are performed for a single mode and coupled modes for different cases of loads. 


\section{THEORETICAL FORMULATION}

To obtain the mathematical statement of displacements of the advanced beam under the assumptions of the Euler-Bernoulli theory of beams, it is required to define the co-ordinate system for the beam subjected to axial, transverse loads and twisting moment. The axial and transverse deformations are shown in Fig.1. and Fig.2. while Fig.3. shows the torsional displacement of the beam. The following formulations are obtained,

$$
\Delta Y=r \cdot \cos (\beta+\varphi)-r \cdot \cos (\beta)=r \cdot(\cos \beta \cdot \cos \varphi-\sin \beta \cdot \sin \varphi)-r \cdot \cos \beta
$$

For small deformation angle $(\varphi)$,

$$
\cos (\varphi) \cong 1, \quad \sin (\varphi) \cong \varphi
$$

Therefore,

$$
\Delta Y=r \cdot(\cos \beta \cdot 1-\varphi \cdot \sin \beta)-r \cdot \cos \beta=-\varphi \cdot r \cdot \sin (\beta)
$$

Since $Z=r$. $\sin (\beta)$, thus,

$$
\Delta \mathbf{Y}=-\mathbf{Z} \cdot \varphi
$$

Similarly,

$$
\begin{array}{r}
\Delta Z=r \cdot \sin (\beta+\varphi)-r \cdot \sin (\beta)=r \cdot(\cos \beta \cdot \sin \varphi+\sin \beta \cdot \cos \varphi)-r \cdot \sin \beta \\
\Delta Z=r \cdot(\varphi \cdot \cos \beta-\sin \beta \cdot 1)-r \cdot \sin \beta=\varphi \cdot r \cdot \cos (\beta) \\
\Delta Z=Y \cdot \varphi
\end{array}
$$

The warping effect is appeared in Fig.4. and the warping function must satisfy two conditions:

$$
\begin{gathered}
\nabla^{2} \lambda=\frac{\partial^{2} \lambda}{\partial \mathbf{y}^{2}}+\frac{\partial^{2} \lambda}{\partial \mathbf{z}^{2}}=0 \\
\left(\frac{\partial \lambda}{\partial \mathbf{y}}-\mathbf{z}\right) \cdot \frac{\mathbf{d z}}{\mathbf{d s}}-\left(\frac{\partial \lambda}{\partial \mathbf{z}}-\mathbf{y}\right) \cdot \frac{\mathbf{d y}}{\mathbf{d s}}=\mathbf{0}
\end{gathered}
$$

From these conditions, warping function can be written as;

$$
\lambda=\mathbf{K}_{\text {warping }} \cdot \mathbf{y} \cdot \mathbf{z}
$$

Where $K_{\text {warping }}$ is the cross-sectional warping coefficient. For Aluminum rectangular beam $K_{\text {warping }}=1$

Thus, the assumed displacement field equations based on the classical lamination theory in the $\mathrm{x}, \mathrm{y}$, and $\mathrm{z}$ directions are,

$$
\begin{aligned}
& U(x)=U_{s}(x)-z \cdot \frac{\partial W(x)}{\partial x} \\
& V(x)=-z \cdot \phi(x) \\
& W(x)=\mathbf{w}(x)+y \cdot \phi(x)
\end{aligned}
$$


Where $U_{s}(x)$ is the mid plane axial displacement, $w(x)$ is the bending displacement due to transverse load and $\varphi(x)$ is the twist angle.

The warping function will appear when differentiating the displacement in z-direction,

$\mathbf{z} \cdot \frac{\partial \mathbf{W}(\mathbf{x})}{\partial \mathbf{x}}=\mathbf{z} \cdot \frac{\partial}{\partial \mathbf{x}}(\mathbf{w}(\mathbf{x})+\mathbf{y} \cdot \phi(\mathbf{x}))=\mathbf{z} \cdot \frac{\partial \mathbf{w}(\mathbf{x})}{\partial \mathbf{x}}+\mathbf{z} \cdot \mathbf{y} \cdot \frac{\partial \phi(\mathbf{x})}{\partial \mathbf{x}}=\mathbf{z} \cdot \frac{\partial \mathbf{w}(\mathbf{x})}{\partial \mathbf{x}}+\lambda \cdot \frac{\partial \phi(\mathbf{x})}{\partial \mathbf{x}}$

\section{STRAIN-DISPLACEMENT RELATIONS}

The normal strain $(\varepsilon)$ and shear strain $(\gamma)$ can be obtained simply by differentiating the displacements, equations as;

$$
\begin{array}{lll}
\varepsilon_{\mathrm{xx}}=\frac{\partial \mathbf{U}(\mathbf{x})}{\partial \mathbf{x}} & \varepsilon_{\mathrm{yy}}=\frac{\partial \mathbf{V}(\mathbf{x})}{\partial \mathbf{y}} & \varepsilon_{\mathrm{zz}}=\frac{\partial \mathbf{W}(\mathbf{x})}{\partial \mathbf{z}} \\
\gamma_{\mathrm{zx}}=\frac{\partial \mathbf{U}(\mathbf{x})}{\partial \mathbf{z}}+\frac{\partial \mathbf{W}(\mathbf{x})}{\partial \mathbf{x}} & \gamma_{\mathrm{zy}}=\frac{\partial \mathbf{V}(\mathbf{x})}{\partial \mathbf{z}}+\frac{\partial \mathbf{W}(\mathbf{x})}{\partial \mathbf{y}} & \gamma_{\mathrm{xy}}=\frac{\partial \mathbf{U}(\mathbf{x})}{\partial \mathbf{y}}+\frac{\partial \mathbf{V}(\mathbf{x})}{\partial \mathbf{x}}
\end{array}
$$

By substituting equation (6) into eqn. (8), one can obtain;

$$
\begin{array}{llc}
\varepsilon_{\mathrm{xx}}=\frac{\partial \mathbf{U}_{\mathrm{s}}(\mathbf{x})}{\partial \mathbf{x}}-\mathbf{z} \cdot \frac{\partial^{2} \mathbf{w}(\mathbf{x})}{\partial \mathbf{x}^{2}}-\mathbf{y} \cdot \mathbf{z} \cdot \frac{\partial^{2} \varphi(\mathbf{x})}{\partial \mathbf{x}^{2}} & \varepsilon_{\mathrm{yy}}=0 \quad \varepsilon_{\mathrm{zz}}=0 \\
\gamma_{\mathrm{zx}}=0 & \gamma_{\mathrm{zy}}=0 & \gamma_{\mathrm{xy}}=-2 \mathrm{z} \cdot \frac{\partial \varphi(\mathbf{x})}{\partial \mathbf{x}}
\end{array}
$$

\section{VARIATIONAL FORMULATION}

The element equations of motion can be detected by means of variational approach by writing the Kinetic energy, Potential energy, and the Virtual work expressions in term of the nodal coordinates.

Using the principle of total strain energy [13],

$$
\delta \mathbf{U}_{i}=\delta \mathbf{W}_{e}
$$

Equation (10) will be expanded as follows,

$$
\begin{aligned}
\int_{V}\left(\sigma_{x x} \delta \varepsilon_{x x}+\sigma_{y y} \delta \varepsilon_{y y}+\sigma_{z z} \delta \varepsilon_{z z}+\tau_{z x} \delta \gamma_{z x}+\tau_{z y} \delta \gamma_{z y}+\tau_{x y} \delta \gamma_{x y}\right) \cdot d V= \\
\int_{0}^{h} P(x) \cdot \frac{\partial U_{s}(x)}{\partial x} \cdot d x+\int_{0}^{h} \Omega(x) \cdot \frac{\partial w(x)}{\partial x} \cdot d x+\int_{0}^{h} T(x) \cdot \frac{\partial \varphi(x)}{\partial x} \cdot d x
\end{aligned}
$$

By using Equation (9); the energy equation (11) can be simplified as, 


$$
\int_{V}\left(\sigma_{x x} \delta \varepsilon_{x x}+\tau_{x y} \delta \gamma_{x y}\right) \cdot d V=\int_{0}^{h} P(x) \cdot \frac{\partial U_{s}(x)}{\partial x} \cdot d x+\int_{0}^{h} \Omega(x) \cdot \frac{\partial w(x)}{\partial x} \cdot d x+\int_{0}^{h} T(x) \cdot \frac{\partial \varphi(x)}{\partial x} \cdot d x
$$

Where $P(x)$ is the axial load per unit length $[N / m], \Omega(x)$ is the transverse load per unit length $[N / m]$, and $T(x)$ is the twisting moment per unit length $[N] . P(x), \Omega(x)$ and $T(x)$ are assumed to be constant along the span $x$, then equation (12) can be written as,

$$
\int_{V}\left(\delta \varepsilon_{x x} \cdot E \cdot \varepsilon_{x x}+\delta \gamma_{x y} \cdot G \cdot \gamma_{x y}\right) \cdot d \mathbf{V}=P \int_{0}^{h} \frac{\partial U_{s}(x)}{\partial \mathbf{x}} \cdot d \mathbf{d x}+\Omega \int_{0}^{h} \frac{\partial \mathbf{w}(\mathbf{x})}{\partial \mathbf{x}} \cdot \mathbf{d x}+\mathrm{T} \int_{0}^{\mathrm{h}} \frac{\partial \varphi(\mathbf{x})}{\partial \mathbf{x}} \cdot \mathbf{d x}
$$

The element mass matrix is obtained using the kinetic energy term as;

$$
\begin{aligned}
& T(t)=\frac{1}{2} \cdot \int_{0}^{L} m(x) \cdot v^{2} \cdot d x \\
& =\frac{1}{2} \int_{-L / 2}^{L / 2}\left(m(x) \cdot \dot{U}^{2}+m(x) \cdot \dot{w}^{2}+\rho \cdot I_{x}(x) \cdot \dot{\varphi}^{2}\right) \cdot d x
\end{aligned}
$$

Where $\rho$ is the density of the material, $v$ is the velocity (displacement differentiation), and $m(x)$ is the mass per unit length of the beam.

\section{FINITE ELEMENT MODELING}

The shape functions of the axial deformation $U_{s}(x)$ [14], can be obtained from the differential equation for the axial deformation as,

$$
E \cdot A \cdot \frac{\partial^{2} U_{s}(x)}{\partial x^{2}}=0 \quad 0<x<h
$$

By integrating eqn. (16) twice, and applying the boundary conditions, we obtain the expression for the axial displacement,

$$
\begin{aligned}
& U_{s}(x)=\left(1-\frac{x}{h}\right) \cdot u_{1}+\frac{x}{h} \cdot u_{2} \\
& f_{\text {axial }}=\left[\left(1-\frac{x}{h}\right) \frac{x}{h}\right]
\end{aligned}
$$

The shape function of transverse deformation $w(x)$ [13], is obtained from the differential equation for the transverse displacement as,

$$
E \cdot I \cdot \frac{\partial^{4} \mathbf{w}(\mathbf{x})}{\partial \mathbf{x}^{4}}=0 \quad 0<\mathbf{x}<\mathbf{h}
$$


By integrating eqn. (19) four times and applying the boundary conditions we obtain the expression for the transverse deformation,

$$
\begin{gathered}
w(x)=\left[1-3\left(\frac{x}{h}\right)^{2}+2\left(\frac{x}{h}\right)^{3}\right] \cdot w_{1}+\left[\frac{x}{h}-2\left(\frac{x}{h}\right)^{2}+\left(\frac{x}{h}\right)^{3}\right] \cdot h \theta_{1} \\
+\left[3\left(\frac{x}{h}\right)^{2}-2\left(\frac{x}{h}\right)^{3}\right] \cdot w_{2}+\left[-\left(\frac{x}{h}\right)^{2}+\left(\frac{x}{h}\right)^{3}\right] \cdot h \theta_{2} \\
f_{\text {bending }}=\left[\left[1-3\left(\frac{x}{h}\right)^{2}+2\left(\frac{x}{h}\right)^{3}\right]\left[\frac{x}{h}-2\left(\frac{x}{h}\right)^{2}+\left(\frac{x}{h}\right)^{3}\right]\left[3\left(\frac{x}{h}\right)^{2}-2\left(\frac{x}{h}\right)^{3}\right]\left[-\left(\frac{x}{h}\right)^{2}+\left(\frac{x}{h}\right)^{3}\right]\right]
\end{gathered}
$$

The shape function of the Torsion displacements $\varphi(\mathrm{x})$, can be obtained as,

$$
\varphi(x)=c_{1} x^{2}+c_{2} x+c_{3}
$$

By applying boundary conditions to eqn (22), for the three nodal displacements on beam element,

$$
\varphi(0)=\varphi_{1} \quad \varphi(\mathrm{h} / 2)=\varphi_{2} \quad \varphi(\mathrm{h})=\varphi_{3}
$$

By substituting eqn. (23) into eqn. (22), the expression for the torsional displacement is obtained as,

$$
\begin{aligned}
& \varphi(x)=\left[2\left(\frac{x}{h}\right)^{2}-3\left(\frac{x}{h}\right)+1\right] \cdot \varphi_{1}+\left[-4\left(\frac{x}{h}\right)^{2}+4\left(\frac{x}{h}\right)\right] \cdot \varphi_{2}+\left[2\left(\frac{x}{h}\right)^{2}-\left(\frac{x}{h}\right)\right] \cdot \varphi_{3} \\
& f_{\text {torsion }}=\left[\left[2\left(\frac{x}{h}\right)^{2}-3\left(\frac{x}{h}\right)+1\right]\left[-4\left(\frac{x}{h}\right)^{2}+4\left(\frac{x}{h}\right)\right]\left[2\left(\frac{x}{h}\right)^{2}-\left(\frac{x}{h}\right)\right]\right]
\end{aligned}
$$

Defining the Nodal displacements for axial, bending and torsion displacements to be " $u_{1}, u_{3}$ ", " $w_{1}, \theta_{1}, w_{3}, \theta_{3}$ " and " $\varphi_{1}, \varphi_{2}, \varphi_{3}$ " respectively, which are illustrated in the Fig.5. By inserting the shape functions into the displacement equations (6), the displacement equation can be written as,

$$
\begin{aligned}
& U(x)=f_{x} \cdot\left[q_{i}\right]=(1-s) u_{1}-\frac{z}{h}\left(-6 s+6 s^{2}\right) w_{1}-z\left(1-4 s+3 s^{2}\right) \theta_{1}-\frac{y \cdot z}{h}(4 s-3) \phi_{1}+(s) u_{3} \\
& -\frac{y \cdot z}{h}(-8 s+4) \phi_{2}-\frac{z}{h}\left(6 s-6 s^{2}\right) w_{3}-z\left(-2 s+3 s^{2}\right) \theta_{3}-\frac{y \cdot z}{h}(4 s-1) \phi_{3} \\
& V(x)=f_{y} \cdot\left[q_{i}\right]=-z\left(2 s^{2}-3 s+1\right) \phi_{1}-z\left(-4 s^{2}+4 s\right) \phi_{2}-z\left(2 s^{2}-s\right) \phi_{3} \\
& W(x)=f_{z} \cdot\left[q_{i}\right]=\left(1-3 s^{2}+2 s^{3}\right) w_{1}+h\left(s-2 s^{2}+s^{3}\right) \theta_{1}+y\left(2 s^{2}-3 s+1\right) \phi_{1}+y\left(-4 s^{2}+4 s\right) \phi_{2} \\
& +\left(3 s^{2}-2 s^{3}\right) w_{3}+h\left(-s^{2}+s^{3}\right) \theta_{3}+y\left(2 s^{2}-s\right) \phi_{3}
\end{aligned}
$$


Where $f_{x}, f_{y}$ and $f_{z}$ are the combined shape function of the axial, bending and torsion displacement equations, and the parameter $\mathrm{s}=\mathrm{x} / \mathrm{h}$

The strain equations can be written as,

$$
\begin{gathered}
\varepsilon_{\mathrm{xx}}=\frac{\partial \mathbf{U}(\mathbf{x})}{\partial \mathbf{x}}=\frac{\partial}{\partial \mathbf{x}}\left(\left\{\mathbf{f}_{\mathbf{x}}\right\} \cdot\{\mathbf{q}\}\right)=\frac{\partial \mathbf{f}_{\mathbf{X}}}{\partial \mathbf{x}} \cdot\{\mathbf{q}\}=\left\{\mathbf{B}_{\mathrm{XX}}\right\} \cdot\{\mathbf{q}\} \\
\gamma_{\mathrm{xy}}=\frac{\partial \mathbf{U}(\mathbf{x})}{\partial \mathbf{y}}+\frac{\partial \mathbf{V}(\mathbf{x})}{\partial \mathbf{x}}=\frac{\partial}{\partial \mathbf{y}}\left(\left\{\mathbf{f}_{\mathrm{X}}\right\} \cdot\{\mathbf{q}\}\right)+\frac{\partial}{\partial \mathbf{x}}\left(\left\{_{\mathbf{f}_{\mathrm{y}}}\right\} \cdot\{\mathbf{q}\}\right)=\left(\frac{\partial \mathbf{f}_{\mathbf{X}}}{\partial \mathbf{y}}+\frac{\partial \mathbf{f}_{\mathrm{y}}}{\partial \mathbf{x}}\right) \cdot\{\mathbf{q}\} \\
=\left(\left\{\mathbf{B}_{\mathrm{YX}}\right\}+\left\{\mathbf{B}_{\mathrm{XY}}\right\}\right) \cdot\{\mathbf{q}\}=\left\{\mathbf{B}_{\mathrm{YXXY}}\right\} \cdot\{\mathbf{q}\}
\end{gathered}
$$

By inserting the strain and perform the integration,

$$
\begin{aligned}
\left\{\mathbf{q}_{i}\right\}^{\top}\left\{\int_{V}\left(\left[\mathbf{B}_{x x}\right]^{\top}[E] \cdot\left[B_{x x}\right]+\left[B_{y x x y}\right]^{\top}[G] \cdot\left[B_{y x x y}\right]\right) \cdot d V\right\} \cdot\left\{q_{i}\right\} \\
=P \cdot \int_{0}^{h} \frac{\partial U_{s}(\mathbf{x})}{\partial \mathbf{x}} \cdot \mathbf{d x}+\Omega \cdot \int_{0}^{h} \frac{\partial \mathbf{w}(\mathbf{x})}{\partial \mathbf{x}} \cdot \mathbf{d x}+\mathbf{T} \cdot \int_{0}^{h} \frac{\partial \varphi(\mathbf{x})}{\partial \mathbf{x}} \cdot \mathbf{d x}
\end{aligned}
$$

The axial, bending and torsion element stiffness matrix is given by,

$$
\left[K_{\text {total }}\right]=\int_{V}\left[B_{x x}\right]^{\top}[E] \cdot\left[B_{x x}\right]+\left[B_{y x x y}\right]^{\top}[G] \cdot\left[B_{y x x y}\right] \cdot d V
$$

Where the individual axial, bending and torsion stiffness matrices is obtained from,

$$
\begin{gathered}
\mathbf{K}_{\text {axial }}=\int_{0}^{h} \mathbf{E} \cdot \mathbf{A}(\mathbf{x}) \cdot\left\{\frac{\partial \mathbf{f}_{\text {axial }}(\mathbf{x})}{\partial \mathbf{x}}\right\} \cdot\left\{\frac{\partial \mathbf{f}_{\text {axial }}(\mathbf{x})}{\partial \mathbf{x}}\right\}^{\top} \mathbf{d x} \\
\mathbf{K}_{\text {bending }}=\int_{0}^{\mathbf{h}} \mathbf{E} \cdot \mathbf{I}_{\mathbf{x}}(\mathbf{x}) \cdot\left\{\frac{\partial^{2} \mathbf{f}_{\text {bending }}(\mathbf{x})}{\partial \mathbf{x}^{2}}\right\} \cdot\left\{\frac{\partial^{2} \mathbf{f}_{\text {bending }}(\mathbf{x})}{\partial \mathbf{x}^{2}}\right\} \mathbf{d} \mathbf{x} \\
\mathbf{K}_{\text {torsion }}=\int_{0}^{\mathbf{h}} \mathbf{G} \cdot \mathbf{J}(\mathbf{x}) \cdot\left\{\frac{\partial \mathbf{f}_{\text {torsion }}(\mathbf{x})}{\partial \mathbf{x}}\right\} \cdot\left\{\frac{\partial \mathbf{f}_{\text {torsion }}(\mathbf{x})}{\partial \mathbf{x}}\right\}^{\top}+\mathbf{E} \cdot \mathbf{I}_{\mathbf{x}}(\mathbf{x}) \cdot\left\{\frac{\partial^{2} \mathbf{f}_{\text {torsion }}(\mathbf{x})}{\partial \mathbf{x}^{2}}\right\} \cdot\left\{\frac{\partial^{2} \mathbf{f}_{\text {torsion }}(\mathbf{x})}{\partial \mathbf{x}^{2}}\right\}^{\top} \mathbf{d} \mathbf{x}
\end{gathered}
$$

And are given in Appendix (A).

By performing the integral in equation (29), and substitute the strain components, the static equilibrium equation takes the form;

$$
\left[\mathrm{K}_{\text {total }}\right] \cdot\left\{\mathbf{q}_{\mathrm{i}}\right\}=[\mathrm{F}]
$$

Where, $\left[K_{\text {total }}\right]$ is the element stiffness matrix, and $F$ is the force vector, which shown in Appendix (B) and (C); respectively.

The element mass matrix is obtained by using the kinetic energy equations (14) and (15) for the axial, bending, and torsion deformation as follows;

For the axial, bending, and torsion mass components 


$$
\begin{aligned}
& \mathbf{m}_{\text {axial }}=\int_{0}^{h} \rho \cdot \mathbf{A} \cdot\left\{\mathbf{f}_{\text {axial }}(\mathbf{x})\right\} \cdot\left\{\mathbf{f}_{\text {axial }}(\mathbf{x})\right\}^{\top} \mathbf{d x} \\
& \mathbf{m}_{\text {bending }}=\int_{0}^{h} \rho \cdot \mathbf{A} \cdot\left\{\mathbf{f}_{\text {bending }}(\mathbf{x})\right\} \cdot\left\{\mathbf{f}_{\text {bending }}(\mathbf{x})\right\}^{\top} \mathbf{d x} \\
& \mathbf{m}_{\text {torsion }}=\int_{0}^{h} I_{m}(\mathbf{x}) \cdot\left\{\mathbf{f}_{\text {torsion }}(\mathbf{x})\right\} \cdot\left\{\mathbf{f}_{\text {torsion }}(\mathbf{x})\right\}^{\top} \mathbf{d x}
\end{aligned}
$$

Where

$$
I_{m}(x)=\int_{-a / 2}^{a / 2} \int_{-b / 2}^{b / 2} \rho \cdot\left((y)^{2}+(z)^{2}\right) \cdot d y \cdot d z=\frac{\rho \cdot a \cdot b^{3}}{3}+\frac{\rho \cdot b \cdot a^{3}}{3}
$$

The element mass matrices $m_{\text {axial }}, m_{\text {bending, }}$ and $m_{\text {torsion }}$ are given in Appendix (D) The interaction between these elements of masses matrices is derived from the formula,

$$
\mathbf{m}_{\text {total }}=\int_{0}^{h} \rho \cdot\left\{\mathbf{f}_{\text {total }}(\mathbf{x})\right\} \cdot\left\{\mathbf{f}_{\text {total }}(\mathbf{x})\right\}^{\top} \mathbf{d x}
$$

Where $f_{\text {total }}$ explained in appendix $(E)$ and mass matrix explained in Appendix (F).

\section{Equations of Motion}

Assembling the mass, stiffness matrices and the load vector, the global stiffness, mass matrices and load vector are obtained,

$$
[\mathbf{M}] \cdot\lceil\ddot{\mathbf{q}}\rceil+[\mathbf{K}] \cdot\lceil\mathbf{q}\rceil=\lceil\mathbf{F}\rceil
$$

For free vibration analysis, [15]

\section{Eigenvalue problem}

$$
[\mathbf{M}] \cdot\lceil\ddot{\mathbf{q}}\rceil+[\mathbf{K}] \cdot\lceil\mathbf{q}\rceil=\lceil\mathbf{0}\rceil
$$

The problem of determining the value of the square of the natural frequency $\omega^{2}$ is known as the characteristic value of eigenvalue problem, which can be represented as;

$$
-\omega^{2}[\mathbf{M}] \cdot\lceil\mathbf{q}\rceil+[\mathrm{K}] \cdot\lceil\mathbf{q}\rceil=\lceil\mathbf{0}\rceil
$$

By making some manipulation, the solution of the eigenvalue problem is written as,

$$
\left(\left(\omega^{2}\right)_{r} \cdot[D]-[1] \cdot\lceil q\rceil_{r}\right)=0
$$

Where, $\left(\omega^{2}\right)_{r}$ is the system natural frequencies $(r=1,2,3, \ldots n),\lceil\mathbf{u}\rceil_{r}$ represents the eigenvector corresponding to the eigenvalues $\left(\omega^{2}\right)_{r}$, and $[\mathbf{D}]=[\mathbf{K}]^{-1} \cdot[\mathrm{m}]$ 


\section{Checking for normalization and Orthogonality}

The process of adjusting the elements of the natural modes to render their amplitude unique is called normalization which obtained by setting;

$$
\left\{Q_{\text {nor }}\right\}^{\top}[M]\left\{Q_{\text {nor }}\right\}=[1] \quad \text { and } \quad\left\{Q_{\text {nor }}\right\}^{\top}[K]\left\{Q_{\text {nor }}\right\}=\left[\omega^{2}\right]
$$

Where $Q_{\text {nor }} \ldots$ the normalized mode shapes.

While, the Orthogonality with respect to the inertia matrix $[\mathrm{m}]$ and also with respect to the stiffness matrix $[\mathrm{K}]$, is verified when,

$$
\{Q\}_{i}^{\top}[M]\{Q\}_{j}=[0] \quad \text { and }\{Q\}_{i}^{\top}[K]\{Q\}_{j}=[0] \text { and } i \neq j
$$

The vectors then said to be Orthonormal to each other

\section{VALIDATION EXAMPLE}

An Aluminum beam has the following properties is used for validate the model for a static and dynamic response

$$
\begin{array}{lll}
\nu=0.33 \quad ; \rho=2710 \mathrm{~kg} / \mathrm{m}^{3} & ; G=2.59 \times 10^{6} \mathrm{~N} / \mathrm{m}^{2} & ; E=6.89 \times 10^{6} \mathrm{~N} / \mathrm{m}^{2} \\
\mathrm{~L}=0.1524 \mathrm{~m} ; \mathrm{b}=0.0254 \mathrm{~m} & ; \mathrm{a}=0.01524 \mathrm{~m} & ; \mathrm{P}(\mathrm{x})=5 \mathrm{~N} / \mathrm{m} \\
\Omega(\mathrm{x})=-5 \mathrm{~N} / \mathrm{m} ; \mathrm{T}=1 \mathrm{~N} & &
\end{array}
$$

For a cantilever beam under axial bending and torsion loads Fig.7. and Fig.8. shows the axial and bending displacements under the action of multi loads, also Fig.9. shows the torsional displacement versus number of nodes. Fig.10. shows the static deflection in Axial, bending and torsional directions on 3-D drawing.

The bending mode shapes from $1^{\text {st }}$ to $5^{\text {th }}$ are shown in Fig.11. to Fig.15. The bending torsion coupling mode shapes is illustrated from Fig.16. to Fig.20. The coupled natural frequencies is shown for different beam length, 


\begin{tabular}{|c|c|c|c|c|c|}
\hline $\mathrm{L}$ in $\mathrm{mm}$ & $\mathrm{b} / \mathrm{a}$ & Tip deflection in $\mathrm{mm}$ & modes & $\omega_{\text {coupling }}(\mathrm{rad} / \mathrm{sec})$ & $\mathrm{f}_{\text {coupling }}(\mathrm{Hz})$ \\
\hline \multirow{9}{*}{100} & \multirow{3}{*}{1} & \multirow{3}{*}{$-1.0885 \times 10^{-6}$} & 1 & 5107.8 & 812.9316 \\
\hline & & & 2 & 31647.96 & 5036.929 \\
\hline & & & 3 & 87057.39 & 13855.61 \\
\hline & \multirow{3}{*}{2} & \multirow{3}{*}{$-5.4427 \times 10^{-7}$} & 1 & 5109.01 & 813.1242 \\
\hline & & & 2 & 31647.75 & 5036.896 \\
\hline & & & 3 & 87053.7 & 13855.03 \\
\hline & \multirow{3}{*}{3} & \multirow{3}{*}{$-3.6284 \times 10^{-7}$} & 1 & 5107.0 & 812.8043 \\
\hline & & & 2 & 31646.1 & 5036.633 \\
\hline & & & 3 & 87042.91 & 13853.31 \\
\hline \multirow{9}{*}{200} & \multirow{3}{*}{1} & \multirow{3}{*}{$-1.7417 \times 10^{-5}$} & 1 & 1279.85 & 203.6945 \\
\hline & & & 2 & 7991.5 & 1271.887 \\
\hline & & & 3 & 22273.38 & 3544.919 \\
\hline & \multirow{3}{*}{2} & \multirow{3}{*}{$-8.7083 \times 10^{-6}$} & 1 & 1278.67 & 203.5067 \\
\hline & & & 2 & 7991.24 & 1271.845 \\
\hline & & & 3 & 22273.2 & 3544.89 \\
\hline & \multirow{3}{*}{3} & \multirow{3}{*}{$-5.8055 \times 10^{-6}$} & 1 & 1279.1 & 203.5751 \\
\hline & & & 2 & 7991.15 & 1271.831 \\
\hline & & & 3 & 22272.46 & 3544.772 \\
\hline \multirow{9}{*}{300} & \multirow{3}{*}{1} & \multirow{3}{*}{$-8.8171 \times 10^{-5}$} & 1 & 568.48 & 90.4764 \\
\hline & & & 2 & 3558.28 & 566.3179 \\
\hline & & & 3 & 9942.99 & 1582.476 \\
\hline & \multirow{3}{*}{2} & \multirow{3}{*}{$-4.4086 \times 10^{-5}$} & 1 & 568.51 & 90.48118 \\
\hline & & & 2 & 3558.31 & 566.3226 \\
\hline & & & 3 & 9942.92 & 1582.465 \\
\hline & \multirow{3}{*}{3} & \multirow{3}{*}{$-2.9390 \times 10^{-5}$} & 1 & 568.33 & 90.45253 \\
\hline & & & 2 & 3558.28 & 566.3179 \\
\hline & & & 3 & 9942.8 & 1582.446 \\
\hline
\end{tabular}

Table .1. Coupled natural frequencies and tip deflections

\section{CONCLUSION}

A finite element model and a computer code were developed for analyzing a compressor blades subjected to multi mechanical loads. The warping effect was taken into consideration.

The notice torsion variation through the length of the unique element requires an additional node at the middle of the element to give a better presenting of the torsional deformation. The interaction between the axial and bending and torsion terms in the global mass and stiffness matrices are arisen due to the existing coupling. So the most clear torsional modes will be obvious at higher frequencies as shown in graphical representation of the bending-torsional modes.

From this study, it is believed that the computer code based on a finite element formulation could be further developed as a design tool for structures having different layouts and constituent material.

The formulation presented in this paper can be easily extended to anisotropic materials as a 2 nd part of the studies which is in print. 


\section{REFERENCES}

[1] Timoshenko, S.; "Vibration Problems in Engineering", D. Van Nostrand Co., Inc., 2d ed., 1937.

[2] Burgess, C. P.; "The Frequencies of Cantilever Wings in Beam and Torsional Vibrations", NACA TN 746, 1940.

[3] Boukidis, N. A., and Ruggiero, R. J.; "An Iterative Method for Determining Dynamic Deflections and Frequencies,"; Jour. Aero. Sci., vol. 11, no. 4, pp. 319328, Oct. 1944.

[4] Duncan, W. J., and Collar, A. R.; "A Method for the Solution of Oscillation Problems by Matrices" Phil. Mag. and Jour. Sci., vol. 17, no. 115, pp. 865-909 ser. 7, May 1934.

[5] Osama J. Aldarihem and Robert C. Wetherhold; "Mechanics and control of coupled bending and twisting vibration of laminated beams", Smart Mater. Struct. 123-133, 1997

[6] Sakawa Y. and Luo Z. H.; "Modeling and control of coupled bending and torsional vibrations of flexible beams"; IEEE Trans. Automatic Control 34, pp. 970-7, 1989.

[7] Banks H. T. and Smith C. A.; "Modeling of coupled bending and torsion in elastic structures"; ASME J. Vibr. Control Mech. Sys.61, pp. 11-20, 1993.

[8] Shen I. Y.; "Bending and torsional vibration control of composite beams through intelligent constrained-layer damping treatments", Smart Mater. Struct.4, pp. 349$55,1995$.

[9] Banerjee J. R. and Williams F. W.; "Coupled bending-torsional dynamic stiffness matrix of an axially loaded Timoshenko beam element"; Int. J. Solid Struct. 31 pp. 749-62, 1994.

[10] Boresi A. P., Schmidt R. J. and Sidebottom O. M.; "Advanced Mechanics of Materials"; New York; Wiley pp 242-9, 1978.

[11] Sankar B. V.; "A beam theory for laminated composite and application to torsion problems"; ASME J. Appl. Mech. 60 pp. 246-9, 1993.

[12] Tsai C. L., Daniel I. M. and Yaniv G.; "Torsional response of rectangular composite laminates"; ASME J. Appl. Mech. 57 pp. 383-7, 1990.

[13] Leonard Meirovitch; "Elements of Vibration Analysis"; Mc-Graw Hill, 1986.

[14] David H. Allen and Walter E. Haisler; "Introduction to Aerospace Structural Analysis"; John Wily \& Sons Inc., 1985.

[15] Irving H. Shams and Clive L. Dym.; "Energy and Finite Element Methods in Structural Mechanics"; Hemisphere Publishing Cor., 1985. 


\section{APPENDIX}

\section{Appendix (A)}

$$
\begin{aligned}
& K_{\text {axial }}=\left[\begin{array}{cc}
\frac{E \cdot A}{L} & -\frac{E \cdot A}{L} \\
-\frac{E \cdot A}{L} & \frac{E \cdot A}{L}
\end{array}\right], \quad K_{\text {bending }}=\left[\begin{array}{cccc}
\frac{12 \cdot E \cdot I}{L^{3}} & \frac{6 \cdot E \cdot I}{L^{2}} & -\frac{12 \cdot E \cdot I}{L^{3}} & \frac{6 \cdot E \cdot I}{L^{2}} \\
\frac{6 \cdot E \cdot I}{L^{3}} & \frac{4 \cdot E \cdot I}{L^{2}} & -\frac{6 \cdot E \cdot I}{L^{2}} & \frac{12 \cdot E \cdot I}{L^{3}} \\
-\frac{12 \cdot E \cdot I}{L^{3}} & -\frac{6 \cdot E \cdot I}{L^{2}} & \frac{12 \cdot E \cdot I}{L^{2}} & \frac{6 \cdot E \cdot I}{L^{2}} \\
\frac{6 \cdot E \cdot I}{L^{2}} & \frac{12 \cdot E \cdot I}{L^{3}} & -\frac{6 \cdot E \cdot I}{L^{2}} & \frac{4 \cdot E \cdot I}{L^{2}}
\end{array}\right] \\
& \mathrm{K}_{\text {torsion }}=\left[\begin{array}{ccc}
\frac{28}{3} \frac{G \cdot J}{\mathrm{~L}} & \frac{-32}{3} \frac{\mathrm{G} \cdot \mathrm{J}}{\mathrm{L}} & \frac{4}{3} \frac{\mathrm{G} \cdot \mathrm{J}}{\mathrm{L}} \\
\frac{-32}{\mathrm{G} \cdot \mathrm{J}} & \frac{64}{\mathrm{~L}} \frac{\mathrm{G} \cdot \mathrm{J}}{\mathrm{L}} & \frac{-32}{\mathrm{G} \cdot \mathrm{G} \cdot \mathrm{J}} \\
\frac{4}{\frac{4}{3} \frac{\mathrm{G} \cdot \mathrm{J}}{\mathrm{L}}} & \frac{-32}{3} \frac{\mathrm{G} \cdot \mathrm{J}}{\mathrm{L}} & \frac{28}{3} \frac{\mathrm{G} \cdot \mathrm{J}}{\mathrm{L}}
\end{array}\right]
\end{aligned}
$$

\section{Appendix (B)}

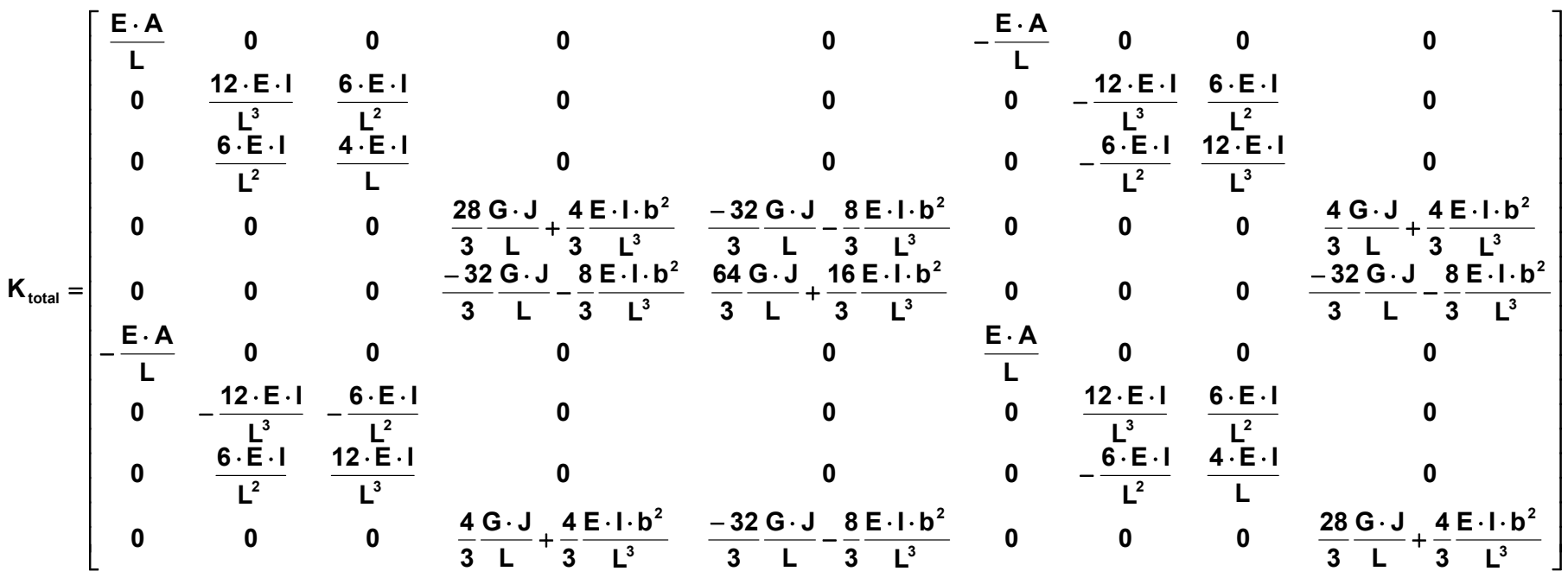

$$
\begin{aligned}
& J=\int_{-a / 2}^{a / 2} \int_{-b / 2}^{b / 2}\left((y-d \lambda / d z)^{2}+(z-d \lambda / d y)^{2}\right) \cdot d y \cdot d z=\frac{b \cdot a^{3}}{12}+\frac{a \cdot b^{3}}{12} \quad I=\int_{-a / 2}^{a / 2} / z^{b / 2} \cdot d y \cdot d z=\frac{b \cdot a^{3}}{12}
\end{aligned}
$$

Appendix (C)

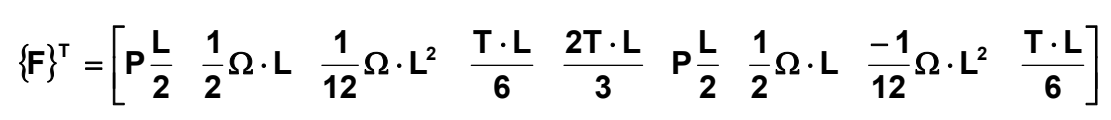

Appendix (D)

$$
m_{\text {axial }}=\left[\begin{array}{ll}
\frac{1}{3} \rho \cdot A \cdot L & \frac{1}{6} \rho \cdot A \cdot L \\
\frac{1}{6} \rho \cdot A \cdot L & \frac{1}{3} \rho \cdot A \cdot L
\end{array}\right]
$$




$$
\begin{aligned}
& \mathrm{m}_{\text {bending }}= {\left[\begin{array}{llll}
\frac{13}{35} \rho \cdot A \cdot L & \frac{11}{210} \rho \cdot A \cdot L^{2} & \frac{9}{70} \rho \cdot A \cdot L & \frac{-13}{420} \rho \cdot A \cdot L^{2} \\
\frac{11}{210} \rho \cdot A \cdot L^{2} & \frac{1}{105} \rho \cdot A \cdot L^{3} & \frac{13}{420} \rho \cdot A \cdot L^{2} & \frac{-1}{140} \rho \cdot A \cdot L^{3} \\
\frac{9}{70} \rho \cdot A \cdot L & \frac{13}{420} \rho \cdot A \cdot L^{2} & \frac{13}{35} \rho \cdot A \cdot L & \frac{-11}{210} \rho \cdot A \cdot L^{2} \\
\frac{-13}{420} \rho \cdot A \cdot L^{2} & \frac{-1}{140} \rho \cdot A \cdot L^{3} & \frac{-11}{210} \rho \cdot A \cdot L^{2} & \frac{1}{105} \rho \cdot A \cdot L^{3}
\end{array}\right] } \\
& m_{\text {torsion }}=\left[\begin{array}{llll}
\frac{2}{15} \cdot I_{m} \cdot L & \frac{1}{15} \cdot I_{m} \cdot L & \frac{-1}{30} \cdot I_{m} \cdot L \\
\frac{2}{15} \cdot I_{m} \cdot L & \frac{8}{15} \cdot I_{m} \cdot L & \frac{1}{15} \cdot I_{m} \cdot L \\
\frac{-1}{30} \cdot I_{m} \cdot L & \frac{1}{15} \cdot I_{m} \cdot L & \frac{2}{15} \cdot I_{m} \cdot L
\end{array}\right]
\end{aligned}
$$

\section{Appendix (E)}

\section{Appendix (F)}

$$
\mathbf{f}_{\text {total }}=\left[\begin{array}{c}
A \cdot \mathbf{f}_{\text {axial }} \\
A \cdot \mathbf{f}_{\text {bending }} \\
I \cdot \mathbf{f}_{\text {torsion }}
\end{array}\right]
$$

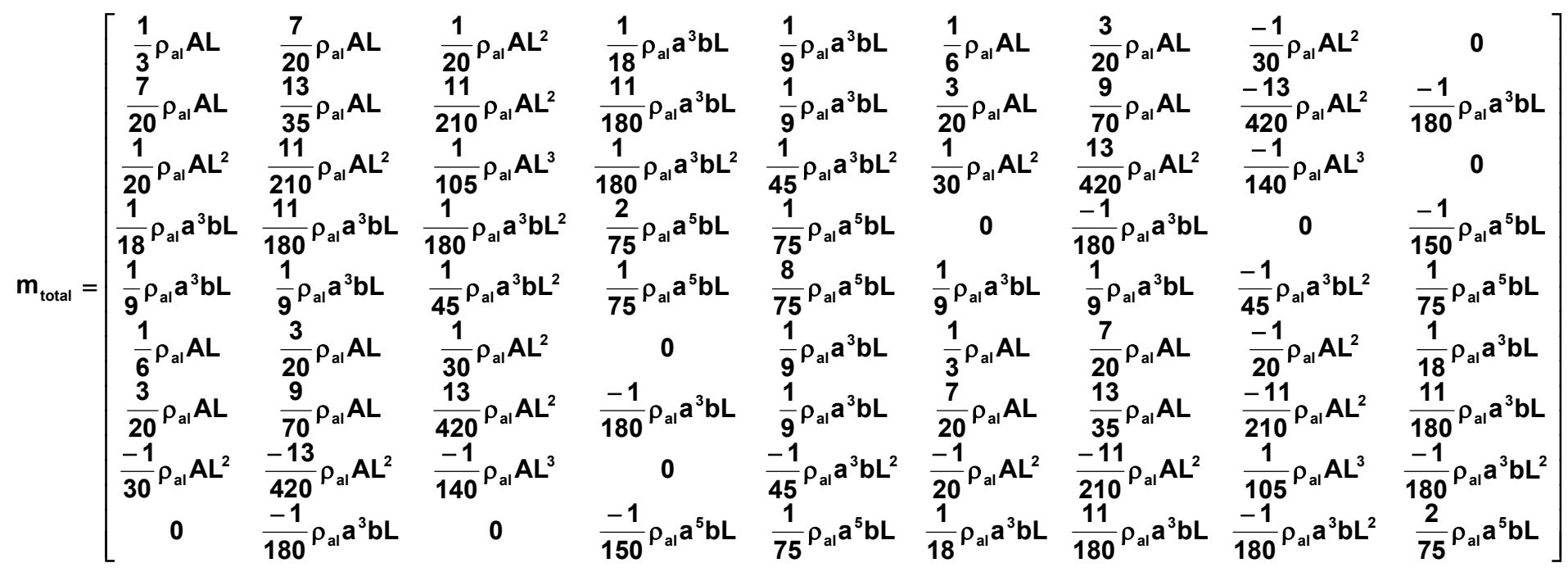




\section{FIGURES}

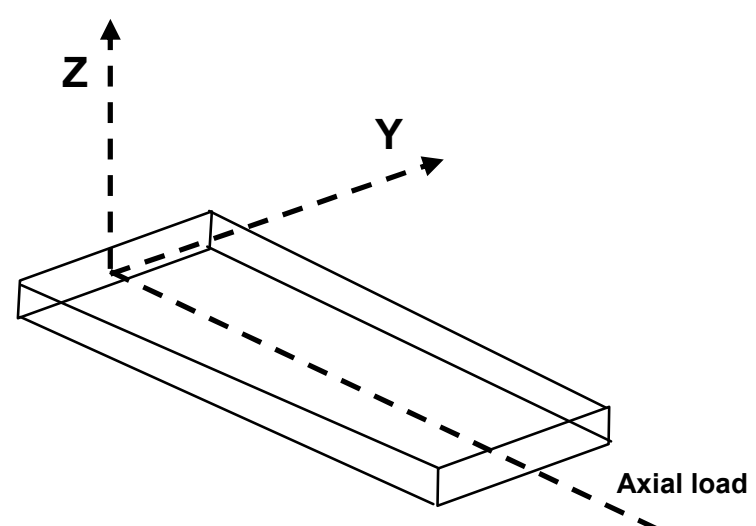

Fig.1. Coordinate system of $\mathbf{X}$ calculated beam with axial load

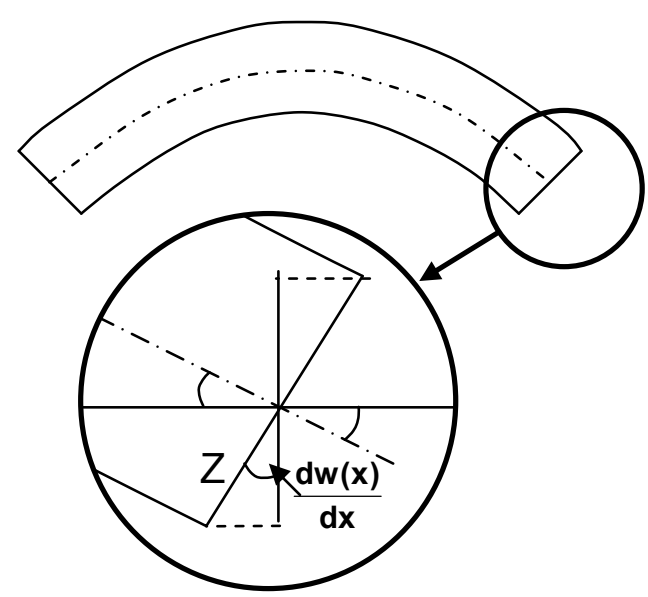

Fig.2. Bending displacement in axial direction

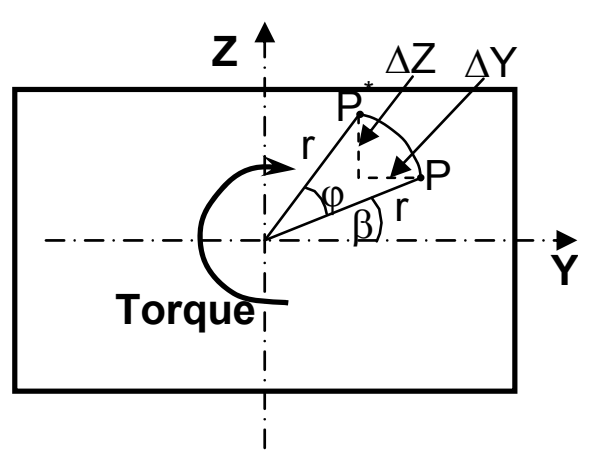

Fig.3. Torsion displacements
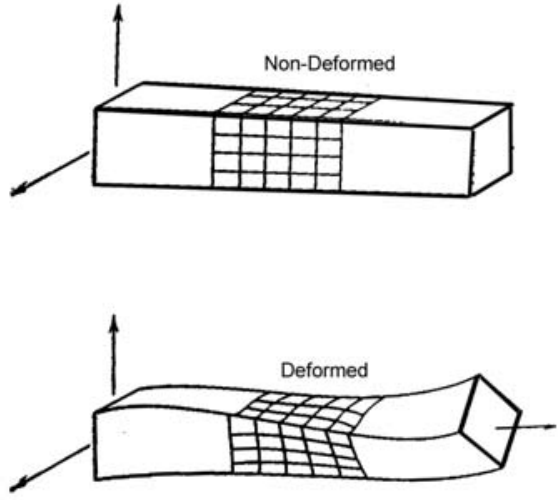

Fig.4. Warping of bar of non-circular cross section Under torsion loading

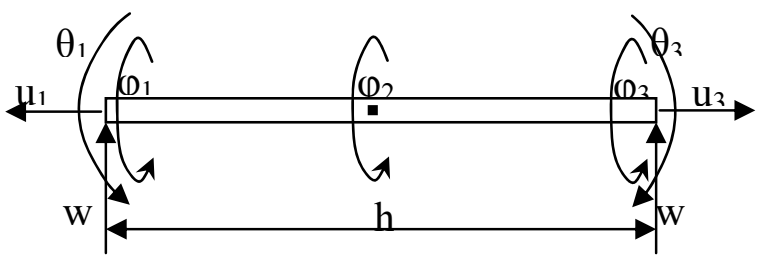

Fig.5. Nodal Displacements of axialbending-torsion Element

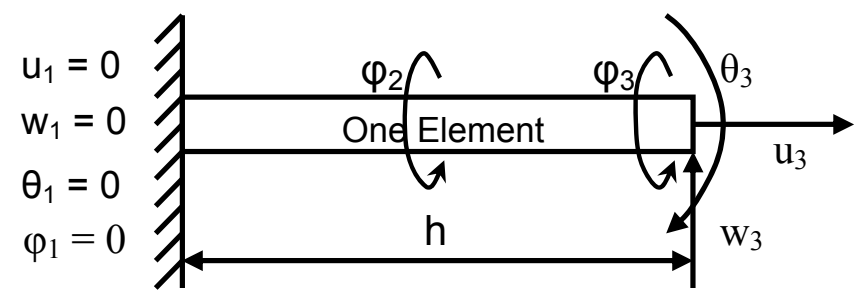

Fig.6. Boundary conditions of Fixed-Free beam 


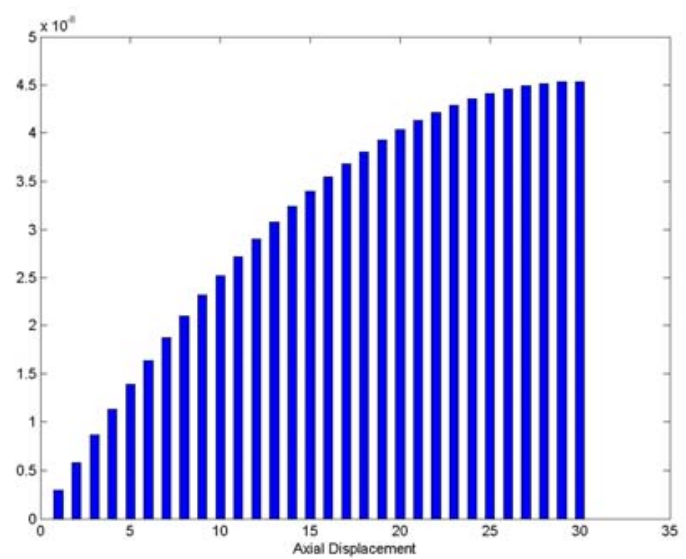

Fig.7. Nodal axial displacements

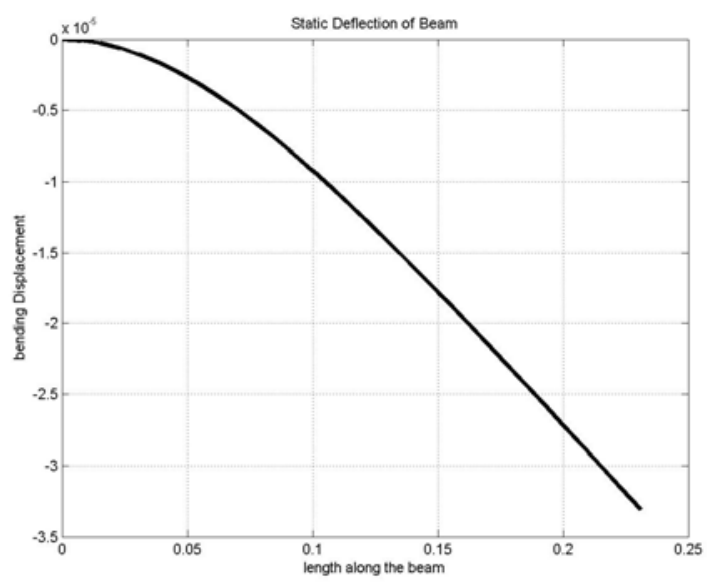

Fig.8. Nodal bending deflection

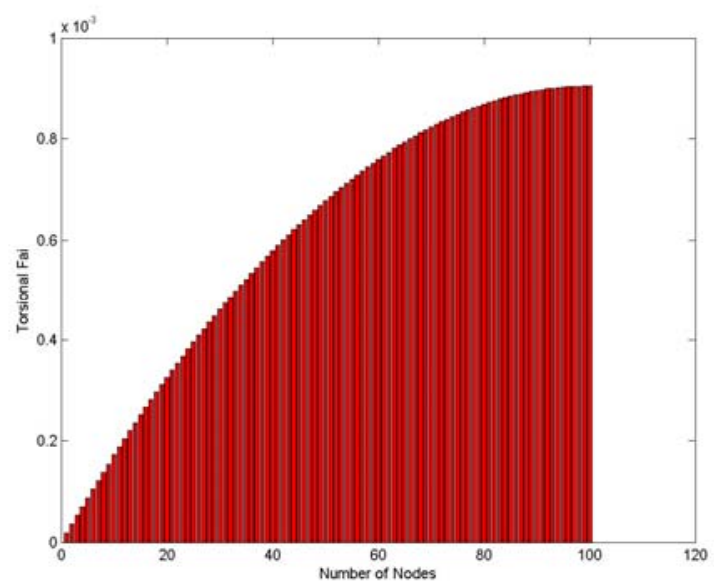

Fig.9. Nodal torsional displacements

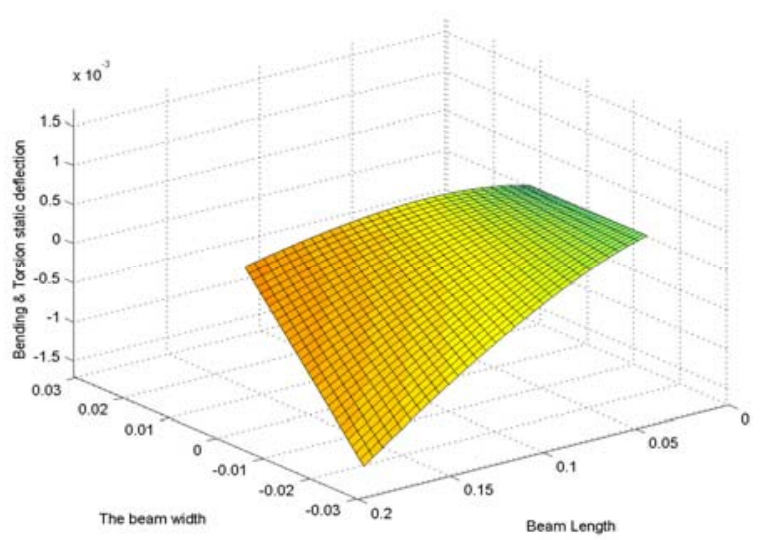

Fig.10. The static coupled bendingtorsion deflection

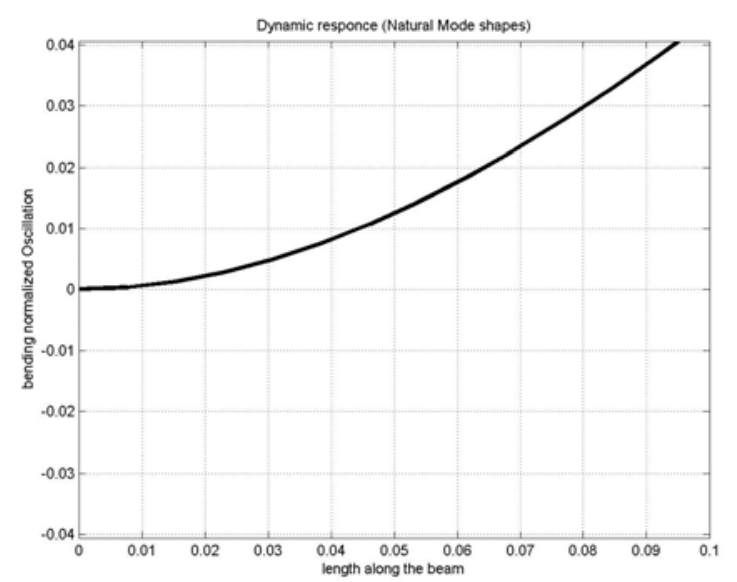

Fig.11. The First bending mode shape

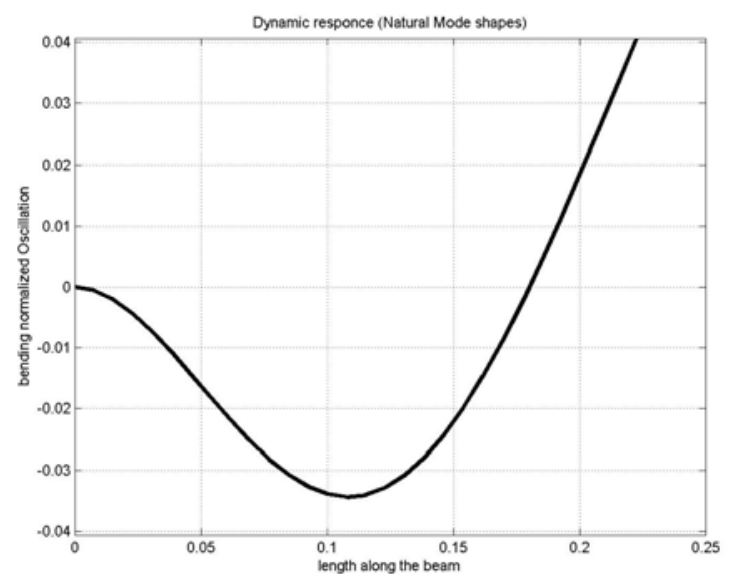

Fig.12. The Second bending mode shape 


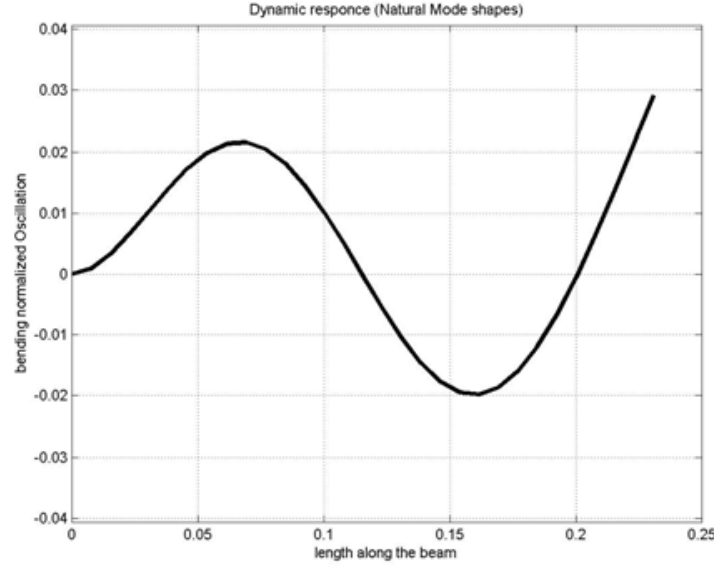

Fig.13. The Third bending mode shape

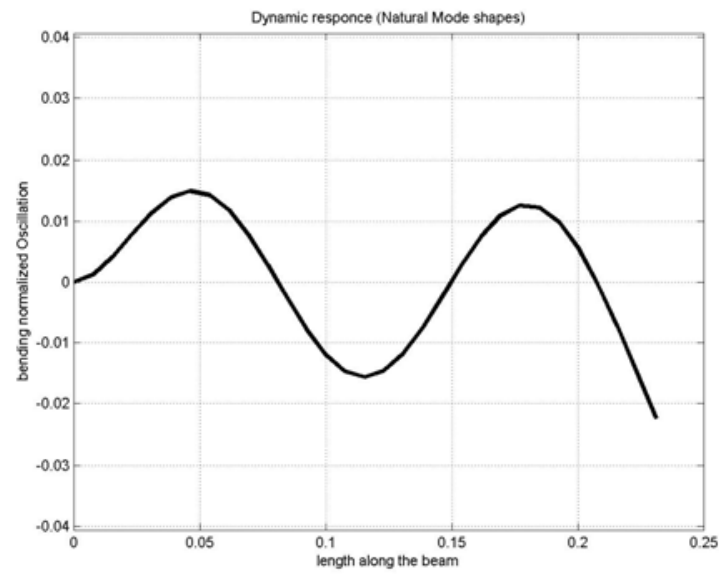

Fig.14. The Fourth bending mode shape

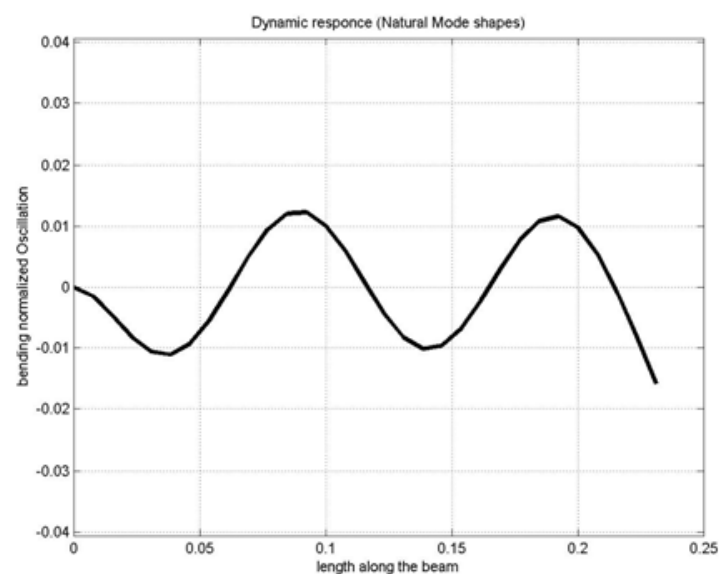

Fig.15. The Fifth bending mode shape

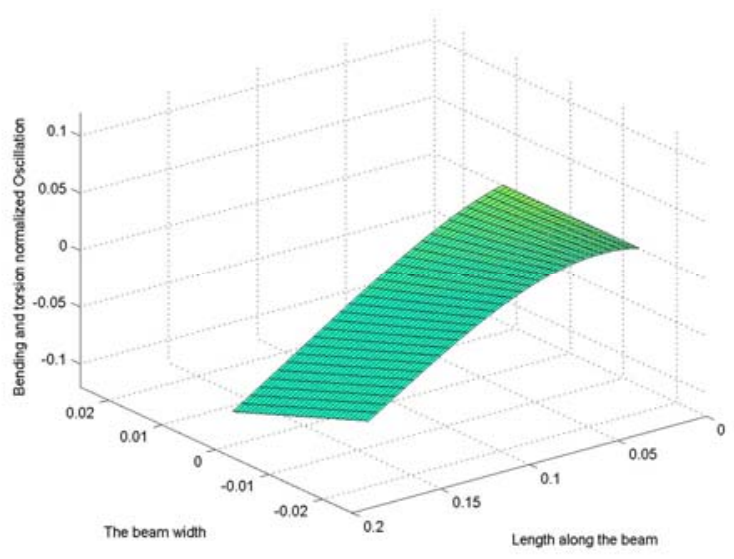

Fig.16. The first bending-torsion coupling mode shape

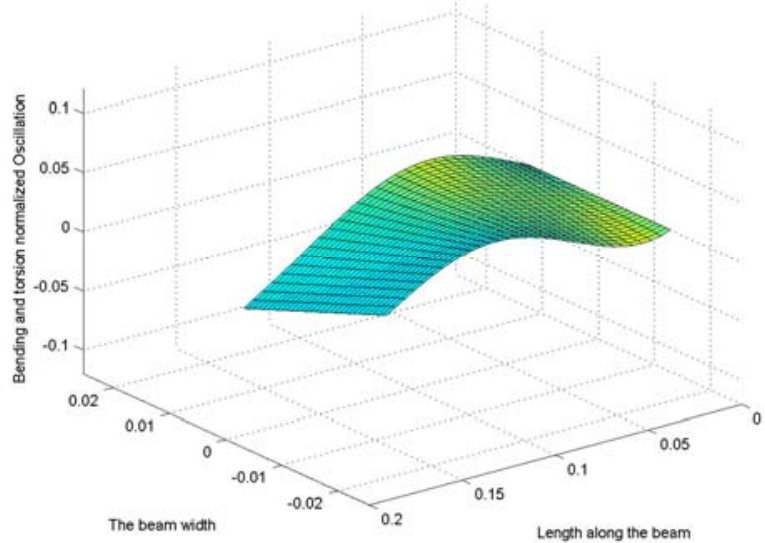

Fig.17. The second bending-torsion coupling mode shape

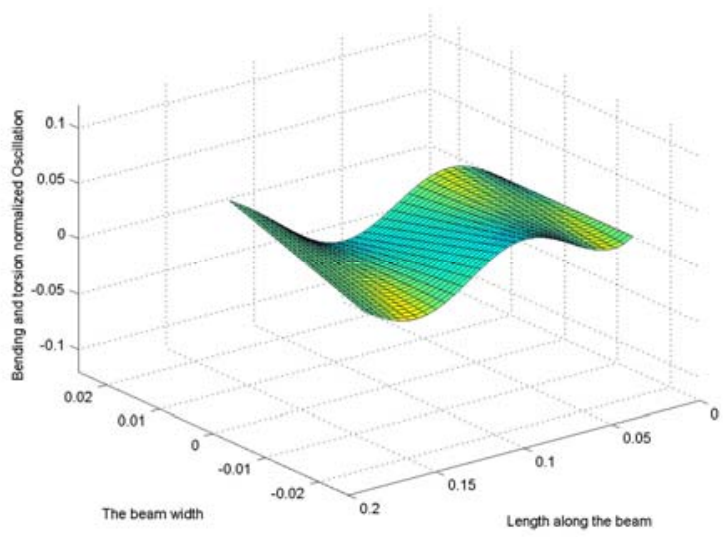

Fig.18. The third bending-torsion coupling mode shape 


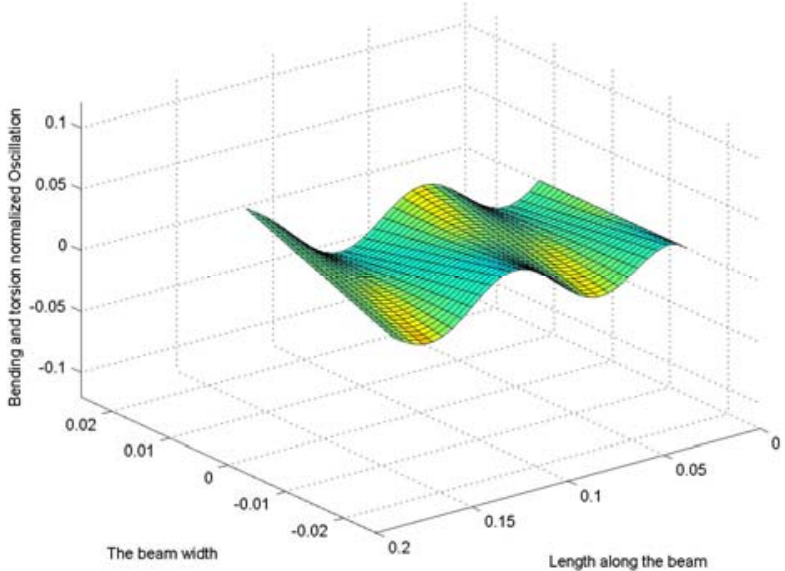

Fig.19. The fourth bending-torsion coupling mode shape

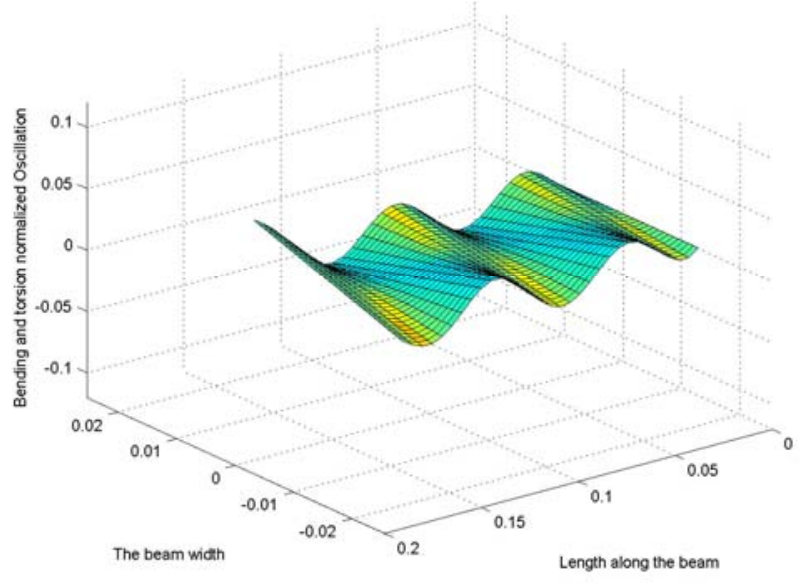

Fig.20. The fifth bending-torsion coupling mode shape 